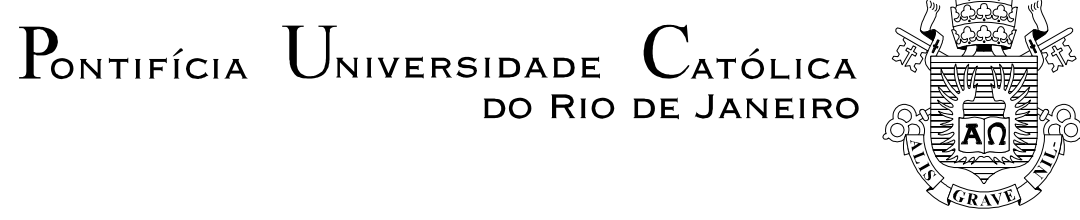

Anderson Pereira

\title{
Projeto ótimo de pórticos planos com restrição à flambagem
}

\begin{abstract}
Dissertação apresentada como requisito parcial para obtenção do grau de Mestre pelo Programa de Pós-graduação em Engenharia Civil do Departamento de Engenharia Civil da PUC-Rio. Área de Concentração: Estruturas.
\end{abstract}

Orientador: Luiz Eloy Vaz Co-orientador: Paulo Batista Gonçalves 


\title{
Pontifícia Universidade Católica $_{\text {a }}$ \\ DO RIO DE JANEIRO

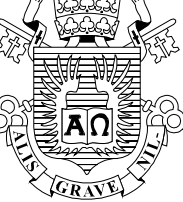

Anderson Pereira

\section{Projeto ótimo de pórticos planos com restrição à flambagem}

\begin{abstract}
Dissertação apresentada como requisito parcial para obtenção do grau de Mestre pelo Programa de Pós-graduação em Engenharia Civil do Departamento de Engenharia Civil do Centro Técnico Científico da PUC-Rio. Aprovada pela Comissão Examinadora abaixo assinada.
\end{abstract}

Prof. Luiz Eloy Vaz

Orientador

Departamento de Engenharia Civil - PUC-Rio

Prof. Paulo Batista Gonçalves

Co-orientador

Departamento de Engenharia Civil - PUC-Rio

Prof. Raul Rosas e Silva

Departamento de Engenharia Civil - PUC-Rio

Profa. Cláudia Ribeiro Eboli

Universidade Federal do Rio de Janeiro

Prof. Ney Augusto Dumont

Departamento de Engenharia Civil - PUC-Rio

Rio de Janeiro, 29 agosto de 2002 
Todos os direitos reservados. É proibida a reprodução total ou parcial do trabalho sem autorização da universidade, do autor e do orientador.

\section{Anderson Pereira}

Graduou-se em Engenharia Civil na UDESC/Joinville (Universidade do Estado de Santa Catarina) em 2000. Interesses acadêmicos em áreas de pesquisa que envolvam otimização de estruturas, programação matemática, análise não-linear e elementos finitos. Atualmente é aluno de doutorado no departamento de engenharia civil da PUC-Rio e pesquisador do Tecgraf - Grupo de tecnologia em computação gráfica.

Ficha Catalográfica

Pereira, Anderson

Projeto ótimo de pórticos planos com restrição à flambagem / Anderson Pereira; orientador: Luiz Eloy Vaz; co-orientador: Paulo Batista Gonçalves. - Rio de Janeiro : PUC, Departamento de Engenharia Civil, 2002.

v., 99 f.: il. ; $29,7 \mathrm{~cm}$

1. Dissertação (mestrado) - Pontifícia Universidade Católica do Rio de Janeiro, Departamento de Engenharia Civil.

Inclui referências bibliográficas

1. Engenharia Civil - Teses. 2. Otimização. 3. Análise Não Linear. 4. Análise de Sensibilidade. 5. Pórticos Planos. 6. Programação Matemática. 7. Instabilidade. 8. Projeto Ótimo. I. Vaz, L. E. (Luiz Eloy); Gonçalves, P. B. (Paulo Batista). II Pontifícia Universidade Católica do Rio de Janeiro. Departamento de Engenharia Civil. III. Título. 
Dedico este trabalho, que se torna pequeno diante do sofrimento que ela tem passado, a minha querida mãe, Idete de Souza Pereira, com muito amor. 


\section{Agradecimentos}

Aos meus orientadores Luiz Eloy Vaz e Paulo Batista Gonçalves pelo estímulo e parceria para a realização deste trabalho.

Aos professores do Departamento de Engenharia Civil da PUC-Rio, por todos os conhecimentos transmitidos durante a pós-graduação.

A Janaína, pelo carinho, compreensão, paciência e constante apoio que fizeram possível a realização deste trabalho.

Ao amigo Sidiclei, a quem responsabilizo pelo inicio da minha vida acadêmica, graças ao seu incentivo e exemplo.

A toda minha família, em especial meus pais, Osvy Manoel Pereira e Idete de Souza Pereira, por todos os gestos de carinho e otimismo.

Aos colegas de república, Alan Wilter S. da Silva, Antonio Miranda e Ricardo Alexandre de Oliveira Passos pelo convívio saudável e pelo ambiente de estudo.

Aos colegas “ótimos”, Ivy e Sandoval, pelas discussões ao longo deste trabalho.

A Claudia Eboli, pelas aulas sobre o algoritmo de Han-Powell.

A todos os amigos da PUC, em especial Galvão, Joabson, Antonio Sérgio, Chan, Salete, Walter e Jaqueline.

Aos funcionários da PUC-Rio, em particular a Ana Roxo, Lenilson, Cristiano, Euclides, Haroldo, José Nilson e Evandro.

Ao CAPES, pelo apoio financeiro. 


\section{Resumo}

Pereira, Anderson; Vaz, Luiz Eloy; Gonçalves, Paulo Batista. Projeto ótimo de pórticos planos com restrição à flambagem. Rio de Janeiro, 2002. 99p. Dissertação de Mestrado - Departamento de Engenharia. Pontifícia Universidade Católica do Rio de Janeiro.

O objetivo deste trabalho é apresentar uma formulação e uma correspondente implementação computacional para otimização de dimensões de estruturas evitando os problemas de instabilidade apresentados pela formulação convencional. Para atingir este objetivo, a formulação utilizada considera os efeitos da não-linearidade geométrica no comportamento da estrutura e inclui uma restrição sobre a carga de colapso. Elementos finitos reticulados planos e a formulação Lagrangiana Atualizada forma utilizados para análise de estruturas com comportamento geometricamente não-linear. As varáveis de projeto são as alturas das seções transversais dos elementos. O método de Newton-Raphson é utilizado acoplado a diferentes estratégias de incremento de carga e de iteração, tais como as que utilizam a restrição do comprimento de arco e as baseadas no controle dos deslocamentos generalizados, que permitem a ultrapassagem de pontos críticos que possam existir ao longo da trajetória de equilíbrio. Os algoritmos de programação matemática utilizados neste trabalho empregam os gradientes da função objetivo e das restrições, que são calculados com base nos gradientes das respostas da estrutura. Partindo-se das equações gerais de equilíbrio válidas para qualquer elemento, foram desenvolvidas expressões analíticas aproximadas que permitem o cálculo das sensibilidades em relação as variáveis de projeto aproveitando as características da análise.

\section{Palavras-chave}

Otimização; análise de sensibilidade; programação matemática; projeto ótimo; pórticos planos; análise não-linear; instabilidade. 


\section{Abstract}

Pereira, Anderson; Vaz, Luiz Eloy; Gonçalves, Paulo Batista. Optimal design of planar frames with stability constraints. Rio de Janeiro, 2002. 99p. Msc. Dissertation - Departamento de Engenharia. Pontifícia Universidade Católica do Rio de Janeiro.

The aim of this work is to present a formulation and corresponding computational implementation for the sizing optimization of structures. To achieve this goal, the formulation considers the structural geometric nonlinear behavior and include a constraint related to the collapse load. Plane frame finite elements and Updated Lagrangian approach are used for the geometric nonlinear analysis. The standard Newton-Raphson method, in connection with different load increment strategies and iteration, such as use the arch length method and strategies based on the control of generalized displacements, which allow the algorithm to transpose the critical points that happen to appear along the equilibrium path. The mathematical programming algorithms applied in this work make use of the gradients of the objective function and of the constraints, which depend on the gradients of the structural response. Starting from general equilibrium equations for the Update Lagrangian approach, valid for any finite element, approximate analytical expressions for the sensitivity analysis whit respect of design variables were developed taking advantage of the structural characteristics.

\section{Keywords}

Optimization; sensitivity analysis; mathematical programming; optimal design; planar frames; nonlinear analysis; stability. 


\section{Sumário}

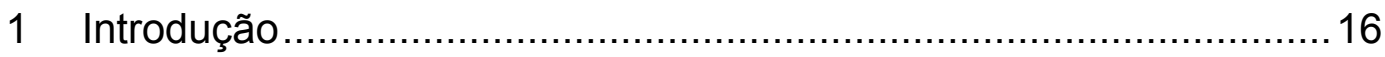

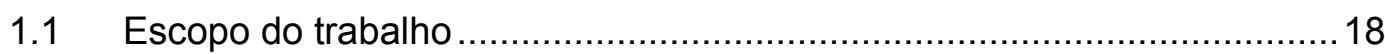

2 Programação Matemática - Princípios Básicos............................. 19

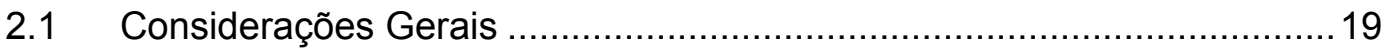

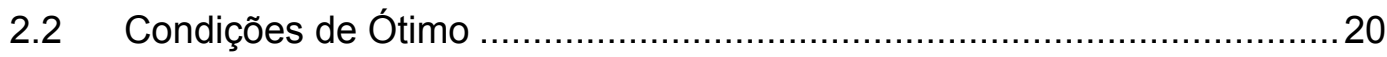

2.3 Forma Geral dos Algoritmos de Otimização ......................................21

2.4 Método de Newton para Problemas de Otimização sem Restrição .........22

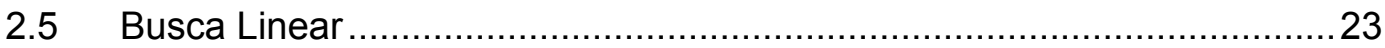

2.6 Programação Quadrática ...........................................................24

2.7 Algoritmo de Han-Powell - Programação Quadrática Seqüencial ............25

2.7.1 Etapas do Algoritmo Não-Linear Han-Powell (PQS)....................................27

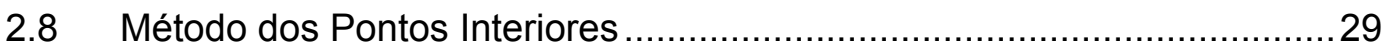

2.8.1 Etapas do Algoritmo de Pontos Interiores (PI) .......................................... 32

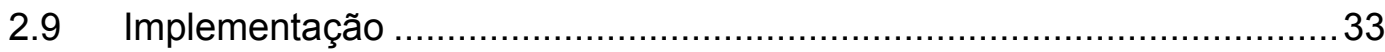

3 Análise Não-Linear Geométrica ........................................... 34

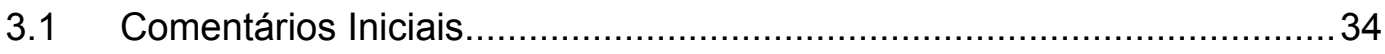

3.2 Comportamento Não-Linear, Análise e Projeto .......................................34

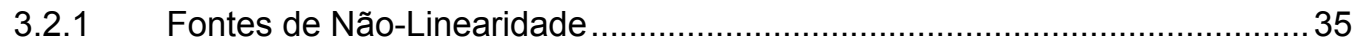

3.3 Formulação para a Análise Não-Linear Geométrica de Estruturas

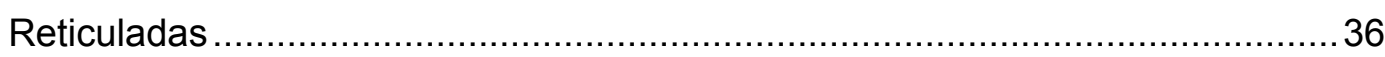

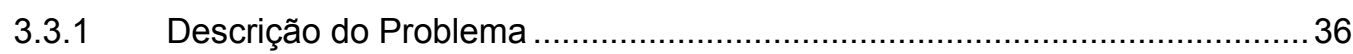

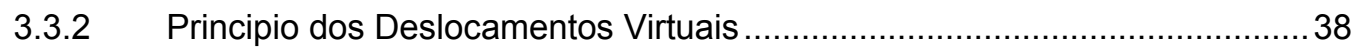


3.3.3 Aplicação a Elementos de Pórtico Plano …................................................ 41

3.3.4 Montagem das Equações da Estrutura ....................................................4 46

3.4 Estratégias de Solução para Problemas Não-Lineares ...........................47

3.4.1 Análise Incremental-Iterativa .............................................................. 48

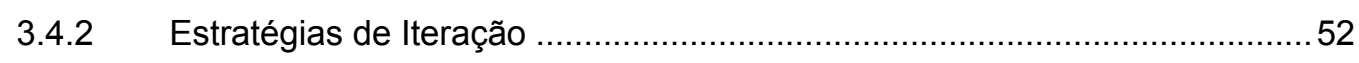

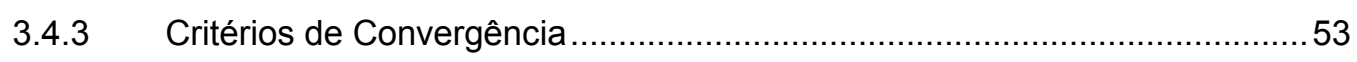

3.4.4 Incremento Automático de Carga............................................................. 55

3.5 Determinação dos Pontos Críticos …............................................ 57

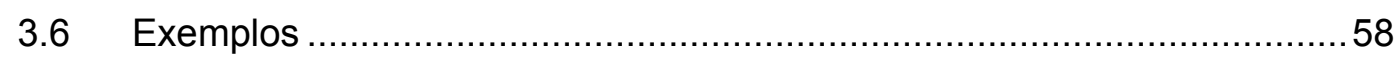

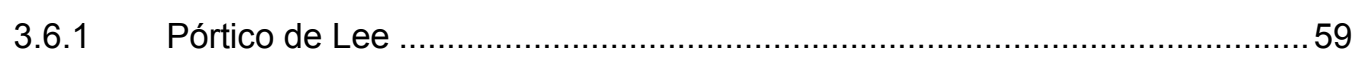

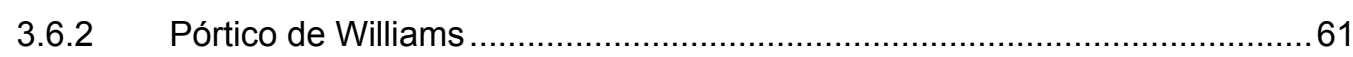

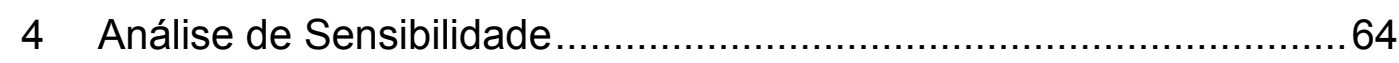

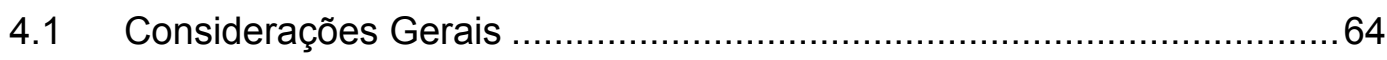

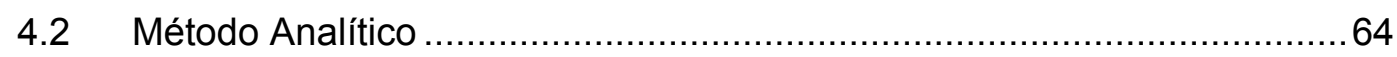

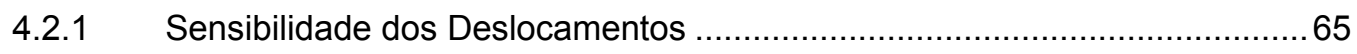

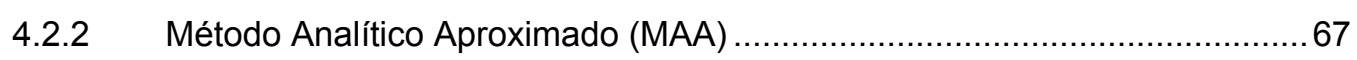

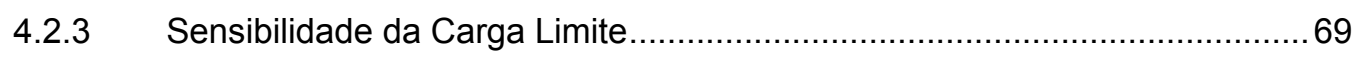

4.3 Método das Diferenças Finitas (MDF) .................................................... 71

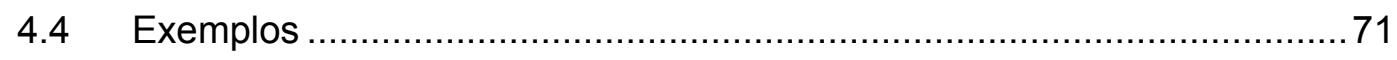

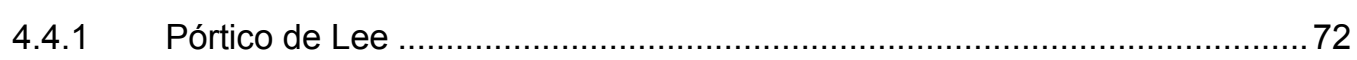

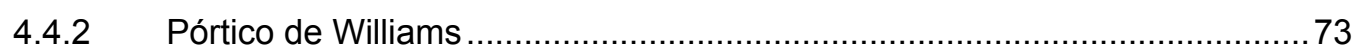

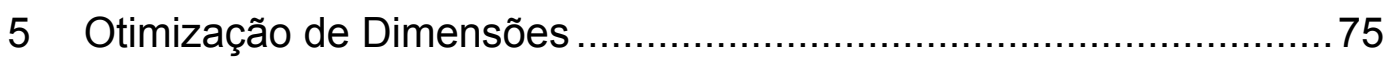

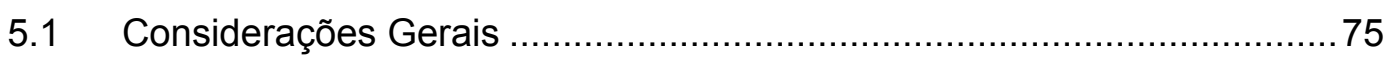

5.2 Metodologia de Otimização .............................................................

5.3 Formulação do problema de Otimização ...........................................77

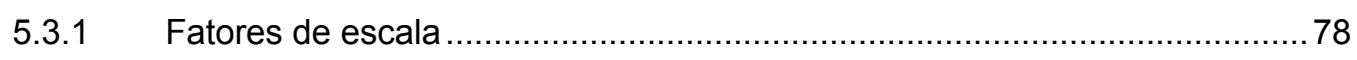

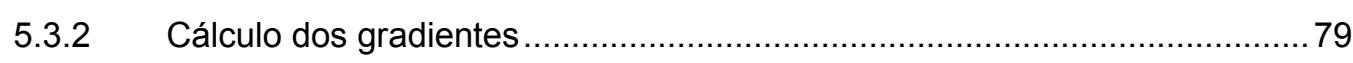

5.4 Implementação da Formulação ……............................................. 81

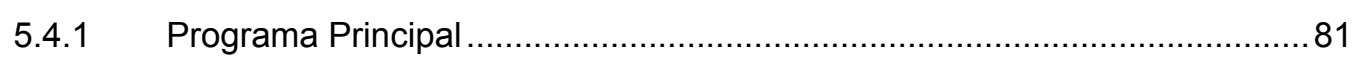




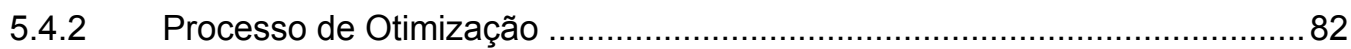

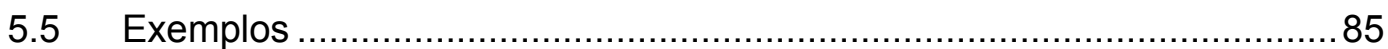

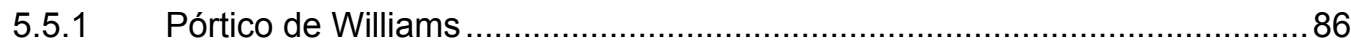

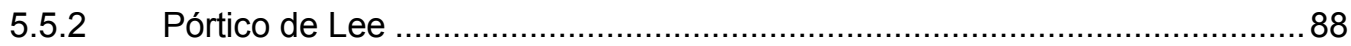

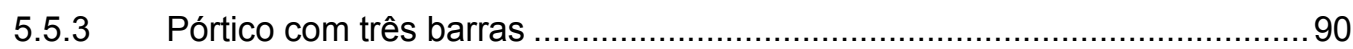

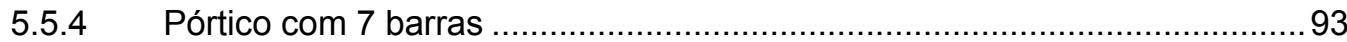

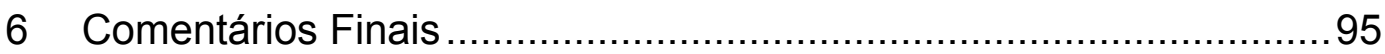

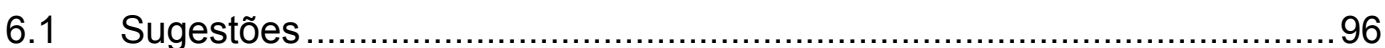

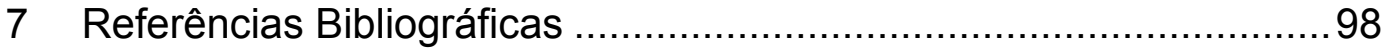




\section{Lista de figuras}

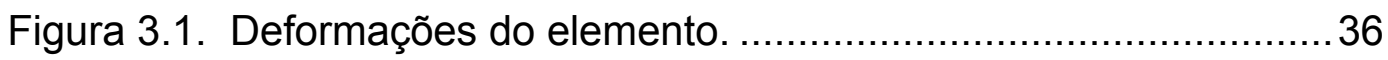

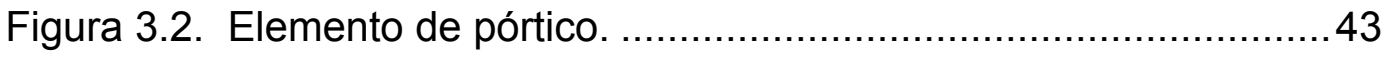

Figura 3.3. Elemento de pórtico. ..................................................... 44

Figura 3.4. Curva carga-deslocamento. ............................................. 48

Figura 3.5. Solução incremental-iterativa: sistema com um grau de

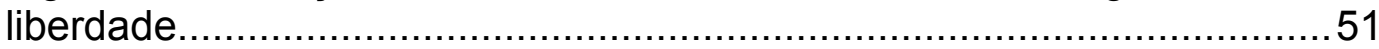

Figura 3.6. Variação do sinal do parâmetro de rigidez generalizado

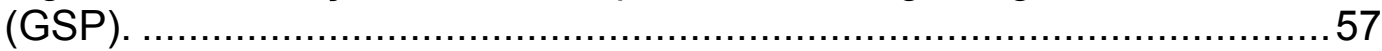

Figura 3.7. Pontos críticos de uma estrutura.......................................58

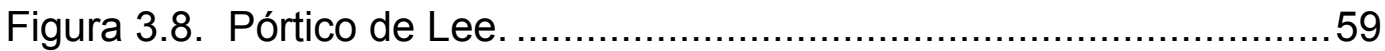

Figura 3.9. Pórtico de Lee - malhas utilizadas.....................................59

Figura 3.10. Pórtico de Lee - curvas de equilíbrio. .................................60

Figura 3.11. Pórtico de Lee - configuração deformada............................60

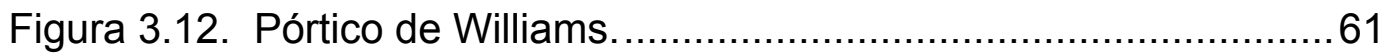

Figura 3.13. Pórtico de Williams - curvas de equilíbrio..........................62

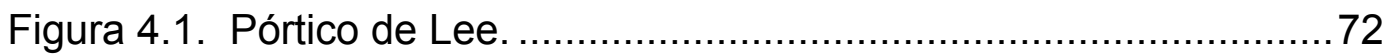

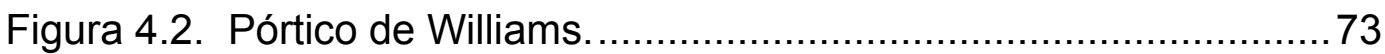

Figura 5.1. Situações encontradas na análise estrutural.........................76

Figura 5.2. Fluxograma do programa principal..................................... 82

Figura 5.3. Forma geral dos algoritmos de PM. .................................. 83

Figura 5.4. Fluxograma da função Análise/Sensibilidade......................... 84

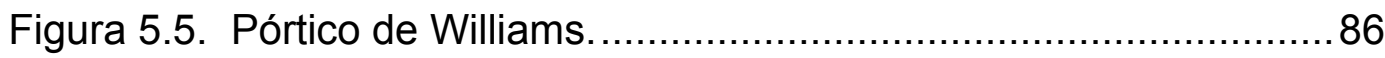

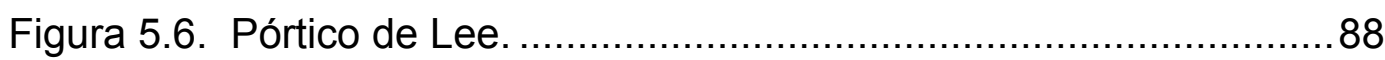

Figura 5.7. Alturas das seções transversais / momento fletor..................89

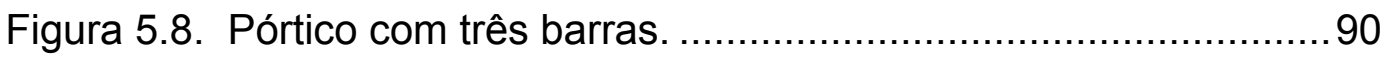

Figura 5.9. Deslocamento horizontal do ponto a para caso linear e não-

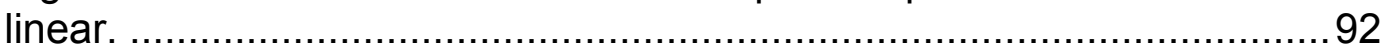

Figura 5.10. Pórtico com 7 barras. .................................................... 93 


\section{Lista de tabelas}

Tabela 2.1 Divisão dos problemas de Programação Matemática 21

Tabela 3.1 Pórtico de Lee - cargas críticas 61

Tabela 3.2 Pórtico de Williams - cargas críticas. 63

Tabela 4.1 Pórtico de Lee - deslocamentos e sensibilidades para $h_{1} \ldots .73$

Tabela 4.2 Pórtico de Lee - deslocamentos e sensibilidades para $h_{2} \ldots .73$

Tabela 4.3 Pórtico de Lee - cargas críticas e sensibilidades .................. 73

Tabela 4.4 Pórtico de Williams - deslocamentos e sensibilidades para $h_{1}$

Tabela 4.5 Pórtico de Williams - cargas críticas e sensibilidades. 74

Tabela 5.1 Valores usuais dos parâmetros. ...................................... 85

Tabela 5.2 Pórtico de Williams - resumo dos resultados ....................... 86

Tabela 5.3 Pórtico de Williams - dimensões finais .............................. 87

Tabela 5.4 Pórtico de Williams - resumo dos resultados ....................... 89

Tabela 5.5 Pórtico de Lee - dimensões finais - pilar ............................. 89

Tabela 5.6 Pórtico de Lee - dimensões finais - viga ............................ 89

Tabela 5.7 Pórtico com três barras - nós restritos ...............................91

Tabela 5.8 Pórtico com três barras - resumo dos resultados ................. 91

Tabela 5.9 Pórtico com três barras - dimensões finais - pilares .............. 91

Tabela 5.10 Pórtico com três barras - dimensões finais - vigas ..............91

Tabela 5.11 Pórtico com 7 barras - resumo dos resultados ...................93

Tabela 5.12 Pórtico com 7 barras - dimensões finais ............................94 


\section{Lista de Símbolos, Siglas e Abreviaturas}

\section{LETRAS ROMANAS}

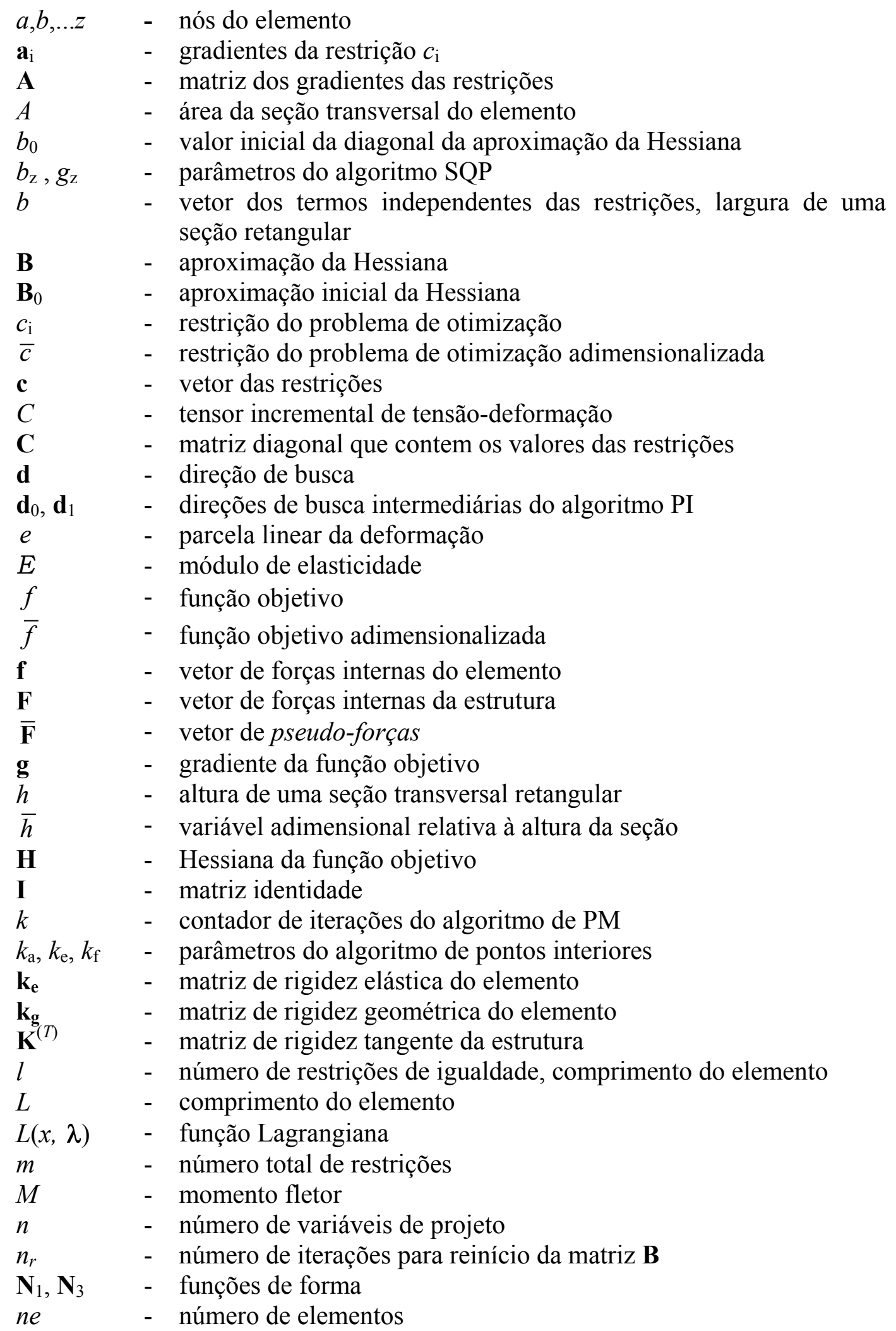




\begin{tabular}{|c|c|}
\hline$n d r$ & - número de deslocamentos restritos \\
\hline$n s e c s$ & - número de grupos de seções transversais \\
\hline$p$ & - ponto material \\
\hline$p(t)$ & - função unidimensional utilizada na busca linear \\
\hline$P$ & - força axial \\
\hline $\mathrm{P}, \mathrm{P} 1, \mathrm{P} 2$ & - forças aplicadas nos elementos \\
\hline $\mathbf{P}$ & - vetor de carregamento externo \\
\hline $\mathbf{P}_{\text {ref }}$ & - vetor de carregamento externo de referência \\
\hline$W$ & - trabalho \\
\hline $\mathbf{W}$ & - Hessiana da função Lagrangiana \\
\hline$w$ & - contador de iterações da análise não-linear \\
\hline q & - $\quad$ intensidade da carga distribuída \\
\hline $\mathbf{q}$ & - $\quad$ vetor da equação de PQ \\
\hline$Q$ & - força cisalhante \\
\hline $\mathbf{Q}$ & - matriz da equação de PQ \\
\hline$y$ & - coordenada cartesiana \\
\hline$r_{\mathrm{i}}$ & - fator de penalidade da busca linear \\
\hline$R$ & - trabalho virtual externo \\
\hline $\mathbf{R}$ & - $\quad$ vetor de forças residuais \\
\hline $\mathfrak{R}$ & - conjunto de números reais \\
\hline$t$ & - tamanho do passo (busca linear) \\
\hline $\mathrm{tol}_{1}, \mathrm{tol}_{2}$ & - tolerâncias do algoritmo PQS \\
\hline tol & - tolerância do algoritmo PI \\
\hline $\mathbf{T}$ & - matriz de rotação \\
\hline$T$ & - $\quad$ tensor de tensões de Cauchy \\
\hline$\tilde{T}$ & - tensor de tensões Piola-Kirchhoff II \\
\hline $\bar{u}, \bar{v}$ & - componentes de deslocamento \\
\hline$u, v$ & - componentes de deslocamento \\
\hline $\mathbf{u}$ & - vetor de deslocamentos nodais \\
\hline $\mathbf{u}_{\mathbf{g}}$ & - $\quad$ parcela de $\mathbf{u}$ referente às forças residuais $\mathbf{g}$ \\
\hline $\mathbf{u}_{\mathbf{r}}$ & - parcela de $\mathbf{u}$ referente às forças de referência $\mathbf{P}_{\text {ref. }}$ \\
\hline $\mathbf{u}_{\mathbf{t}}$ & - vetor dos deslocamentos nodais tangenciais \\
\hline$u_{j, l i m}$ & - valor absoluto admissível para o deslocamento $u_{\mathrm{j}}$ \\
\hline$V$ & - volume \\
\hline$x$ & - vetor das variáveis de projeto, coordenada cartesiana \\
\hline$x, y, z$ & - coordenadas cartesianas \\
\hline$x_{0}$ & - $\quad$ vetor inicial das variáveis de projeto \\
\hline $\bar{x}$ & - vetor das variáveis de projeto adimensionalizada \\
\hline
\end{tabular}

\section{LETRAS GREGAS}

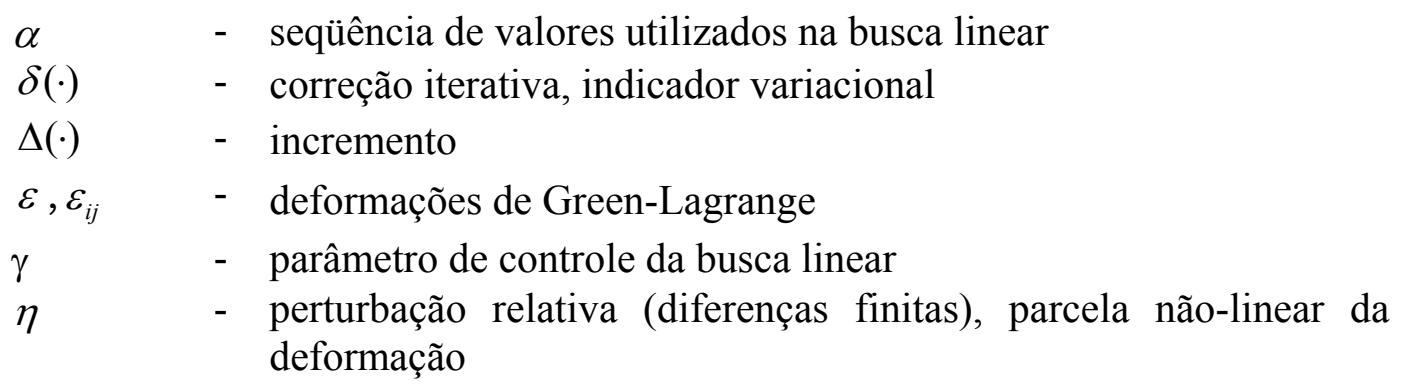


$\varphi \quad-\quad$ autovetor correspondente ao autovalor nulo da matriz de rigidez tangente

$\lambda \quad$ - parâmetro de carga responsável pelo escalonamento de $\mathbf{P}_{\text {ref }}$

$\lambda_{\mathrm{i}} \quad$ - multiplicador de Lagrange da restrição $c_{\mathrm{i}}$

$\lambda \quad-$ vetor dos multiplicadores de Lagrange

$\lambda_{0}, \lambda_{1}, \bar{\lambda}$ - estimativa do vetor dos multiplicadores de Lagrange no algoritmo de pontos interiores

$\bar{\lambda}_{i} \quad-\quad$ valor admissível para a carga crítica

$\bar{\lambda} \quad-$ estimativa do multiplicador de Lagrange no algoritmo de pontos interiores

$\Lambda \quad$ - matriz diagonal contendo os multiplicadores de Lagrange

$\theta \quad$ - ângulo (coordenada polar)

$\rho$ - coeficiente de deflexão da direção de busca

$\tau \quad$ - componentes da tensão de Cauchy

$\zeta \quad$ - tolerância ao resíduo requerida no processo de convergência

$\zeta_{1} \quad$ - fator de convergência baseado em relações de força

$\zeta_{2} \quad$ - fator de convergência baseado em relações de deslocamentos

\section{SOBRESCRITOS E SUBSCRITOS}

${ }^{t}(\cdot) \quad$ - função avaliada no instante $t$

$(\cdot)^{*} \quad$ - função avaliada no ponto crítico

$(\cdot)^{\mathrm{T}} \quad-\quad$ transposta do vetor ou matriz

$(\cdot)_{i j} \quad$ - notação indicial

$(\cdot)^{l} \quad$ - limite inferior da variável de projeto, restrições da carga crítica

$(\cdot)^{u} \quad$ - limite superior da variável de projeto

$(\cdot)^{d} \quad$ - restrições de deslocamento

\section{SigLAS e AbreViaturas}

BFGS - Broyden - Fletcher - Goldfarb - Shanno

GSP - Parâmetro de rigidez generalizado

MAA - Método Analítico Aproximado

MDF - Método das Diferenças Finitas

PM - Programação Matemática

PQ - Programação Quadrática

PQS - Programação Quadrática Seqüencial

RLT - Referencial Lagrangiano Total

RLA - Referencial Lagrangiano Atualizado 